\title{
Successful plasmapheresis treatment of severe hypertriglyceridemia during late pregnancy
}

\author{
Noémi Zsíros, Beáta Kovács, György Paragh, József Balla, Mariann Harangi \\ Department of Internal Medicine, University of Debrecen Faculty of Medicine, Debrecen H-4032, Hungary.
}

Correspondence to: Dr. Mariann Harangi, Department of Internal Medicine, University of Debrecen Faculty of Medicine, Nagyerdei Blvd. 98, Debrecen H-4032, Hungary. E-mail: mharangi@hotmail.com

How to cite this article: Zsíros N, Kovács B, Paragh G, Balla J, Harangi M. Successful plasmapheresis treatment of severe hypertriglyceridemia during late pregnancy. Vessel P/us 2019;3:6. http://dx.doi.org/10.20517/2574-1209.2018.78

Received: 11 Dec 2018 First Decision: 15 Jan 2019 Revised: 22 Jan 2019 Accepted: 23 Jan 2019 Published: 19 Mar 2019

Science Editor: Alexander N. Orekhov Copy Editor: Cai-Hong Wang Production Editor: Huan-Liang Wu

\begin{abstract}
During pregnancy, physiologic hormonal changes provoke a significant increase in triglyceride levels. Genetic abnormalities of triglyceride metabolism and secondary factors may multiply the risk of severe lipid abnormalities. Although severe gestational hypertriglyceridemia can be a life-threatening condition for both mother and fetus, its optimal treatment has not been fully clarified. A 33-year-old woman at 37 weeks of her second pregnancy was admitted to our clinic. Her triglyceride level was $57.8 \mathrm{mmol} / \mathrm{L}$. Abdominal pain, nausea, vomiting or any other complaints were not reported. She kept a fat-restricted diet, however her triglyceride level remained $41 \mathrm{mmol} / \mathrm{L}$. Therefore we decided to perform plasmapheresis with a replacement of human albumin as a colloidal solution. Complications did not occur during the treatment. Plasmapheresis reduced her triglyceride level by $54.1 \%$ (to $18.8 \mathrm{mmol} / \mathrm{L}$ ), and the patient delivered a healthy female neonate at 40 weeks. In case of significantly increased values, plasmapheresis is a fast, effective and safe method for decreasing triglyceride level even in the third trimester.
\end{abstract}

Keywords: Hypertriglyceridemia, pregnancy, plasmapheresis

\section{INTRODUCTION}

Maternal lipids are extremely important for developing a fetus. While cholesterol is a crucial molecule in placental steroid synthesis, fatty acids participate in placental oxidation processes and membrane development ${ }^{[1]}$. In pregnant women, significant alterations in lipid levels are in connection with estrogen, progesterone and human placental lactogen synthesis ${ }^{[2]}$. During normal pregnancy, increased lipolytic activity raises the release of free fatty acid and the production of very low-density lipoprotein (VLDL). 
Besides, the elimination of VLDL is decreased due to the reduced activity of lipoprotein lipase in fatty tissue $^{[3]}$. Eventually, there is a 2-4 fold increase in triglyceride level by third trimester ${ }^{[4]}$.

Genetic abnormalities of triglyceride metabolism can provoke severe hypertriglyceridemia in pregnancy. In familial combined hyperlipidemia and familial hypertriglyceridemia, we see an increased formation of triglyceride-rich lipoproteins (chylomicrons and VLDLs). Ineffective lipolysis of these particles can cause familial chylomicronemia due to the mutations in lipoprotein lipase, apolipoprotein CII or apolipoprotein $\mathrm{AV}^{[5]}$. In familial dysbetalipoproteinemia, apolipoprotein E2/E2 genotype reduces hepatic clearance of chylomicron remnants ${ }^{[5]}$. In addition, secondary factors such as poorly controlled diabetes mellitus (via decreasing the activity of lipoprotein lipase), hypothyroidism, nephrotic syndrome and some medications (glucocorticoids) may aggravate gestational hypertriglyceridemia ${ }^{[6]}$.

Hypertriglyceridemia during pregnancy increases the risk of acute pancreatitis (especially if triglyceride level is above $11.4 \mathrm{mmol} / \mathrm{L}$ ), hyperviscosity syndrome and pre-eclampsia in mother, macrosomia, premature birth or fetal death in fetus ${ }^{[6-9]}$.

At first, treatment of the underlying disease and fat-restricted diet are recommended ${ }^{[6]}$. During pregnancy, lipid-lowering medications (fibrate, nicotinic acid, statin, and ezetimibe) should be stopped due to their possible teratogenic effects ${ }^{[10]}$. Novel lipid-lowering agents including microsomal transfer protein and proprotein convertase subtilisin/kexin type 9 inhibitors are also contraindicated. Therefore, in the case of non-responding severe hypertriglyceridemia plasmapheresis should be considered. This is an extracorporeal procedure, when plasma is separated from the blood and processed to eliminate selective components including triglyceride-rich lipoprotein particles. The treated plasma is then reinfused although, on occasion, it is completely eliminated and replaced by an isovolumetric solution. Most of the studies use plasmapheresis as a treatment of hypertriglyceridemia associated pancreatitis. In some cases, they perform plasmapheresis to prevent pancreatitis. It must be noted that the timing and frequency of plasmapheresis procedures were not uniform in these studies ${ }^{[11-13]}$. During plasmapheresis serum triglyceride level can be decreased by $66 \%-70 \%{ }^{[12,13]}$.

\section{CASE REPORT}

A 33-year-old woman at 37 weeks of her second pregnancy was admitted to our clinic. Her gynecologist indicated routine laboratory examinations, which remained unsuccessful because of lipemic blood sample. Therefore, we checked her triglyceride level and found that it was significantly elevated $(57.8 \mathrm{mmol} / \mathrm{L})$. Abdominal pain, nausea, vomiting or any other complaints were not reported. Based on physical examination, there were no eruptive xanthomas, palmar crease xanthomas or lipaemia retinalis. Her body mass index was within the normal range before pregnancy $(53 \mathrm{~kg}$ ), and her total body weight gain was $9 \mathrm{~kg}$ during pregnancy. In her history, there was no diabetes mellitus or any other secondary causes of hypertriglyceridemia. The diagnosis of gestational diabetes mellitus was evaluated according to the Hungarian national recommendation. We used the modified 1999 WHO recommendation (gestational diabetes mellitus: $75 \mathrm{~g} \mathrm{CH}$ oral glucose tolerance test (OGTT) at 24-28 gestation weeks: fasting plasma glucose $\geq 6.1 \mathrm{mmol} / \mathrm{L}, 120 \mathrm{~min}$ plasma glucose $\geq$ $7.8 \mathrm{mmol} / \mathrm{L})^{[14]}$. Based on the fasting plasma glucose $(5.2 \mathrm{mmol} / \mathrm{L})$ and the OGTT (plasma glucose: $120 \mathrm{~min}$ : $7.1 \mathrm{mmol} / \mathrm{L}$ ) the diagnosis of gestational diabetes mellitus was excluded. In her family history, her mother and grandmother had mixed hyperlipidemia. Her first pregnancy had terminated without any complication however, lipid parameter measurement was not performed. Before her pregnancy, she had not taken any lipid-lowering medications. She had not followed a fat-restricted diet during her pregnancy. Table 1 shows the laboratory parameters before treatment. Markedly elevated triglyceride and total cholesterol levels were detected. Apolipoprotein B100 and lipoprotein (a) levels were also above the upper border of the normal range. Based on the result of lipid electrophoresis, a significant increase in the level of VLDL may cause hypertriglyceridemia. 
Table 1. Laboratory parameters before treatment

\begin{tabular}{llll}
\hline Parameter & Unit & Value & Reference range \\
\hline Total cholesterol & $\mathrm{mmol} / \mathrm{L}$ & $24.7(\mathrm{H})$ & $<5.2$ \\
Triglyceride & $\mathrm{mmol} / \mathrm{L}$ & $41.00(\mathrm{H})$ & $<1.7$ \\
Apolipoprotein A & $\mathrm{g} / \mathrm{L}$ & 1.86 & $>1.15$ \\
Apolipoprotein B100 & $\mathrm{g} / \mathrm{L}$ & $2.25(\mathrm{H})$ & $<1$ \\
Lipoprotein(a) & $\mathrm{mg} / \mathrm{L}$ & $114(\mathrm{H})$ & $<30$ \\
STSH & $\mathrm{mU} / \mathrm{L}$ & 0.978 & $0.300-4.200$ \\
fT3 & $\mathrm{pmol} / \mathrm{L}$ & $1.8(\mathrm{~L})$ & $2.4-6.3$ \\
fT4 & $\mathrm{pmol} / \mathrm{L}$ & $8.2(\mathrm{~L})$ & $12.0-22.0$ \\
CRP & $\mathrm{mg} / \mathrm{L}$ & $8.2(\mathrm{H})$ & $<5.2$ \\
Lipid electrophoresis & & & $15-40$ \\
AlfaLP & $\%$ & $7(\mathrm{~L})$ & $2-31$ \\
PreßLP & $\%$ & $53(\mathrm{H})$ & $42-70$ \\
BetaLP & $\%$ & $34(\mathrm{~L})$ & - \\
Chylomicron & $\%$ & 6 & \\
\hline
\end{tabular}

Her levels of lipase, amylase, transaminases, and inflammatory markers were in the normal range. Abdominal ultrasound showed neither cholelithiasis nor any symptoms of pancreatitis. The development of fetus was appropriate. The patient case was followed by obstetrician, endocrinologist, neonatologist, and dietitian.

She started on a fat-restricted diet $(<20 \%$ of total calories from fat daily) and consumed $15 \mathrm{~g}$ medium-chain triglycerides and $3 \mathrm{~g}$ omega-3-fatty esters daily, but two days later her triglyceride level remained $41 \mathrm{mmol} / \mathrm{L}$. Therefore, she was immediately admitted to our ICU and we decided to perform plasmapheresis using central veins with human albumin infusion as a colloidal solution with one plasma volume exchange and FRESENIUS Com.tec Kabi Therapeutic Plasma Exchange machine.

There were not any complications during plasmapheresis. The patient delivered a healthy female neonate weighing 3,150 g at 40 weeks. One day after delivery her triglyceride level was decreased to $15.68 \mathrm{mmol} / \mathrm{L}$, two more days later it was $7.3 \mathrm{mmol} / \mathrm{L}$ without any further plasmapheresis procedures. The alterations of lipid parameters are shown in Figures 1 and 2.

During lactation, we suggested fat-restricted diet and regular control of triglyceride level. Gene polymorphisms of lipoprotein lipase and Apolipoprotein E were determined. The patient has ApoE 3/3 genotype, therefore, we could exclude the familial dysbetalipoproteinemia as a possible cause. The patient is wild type for two common polymorphisms of LPL (LPL-D9N and LPL-N291S) causing hypertriglyceridemia. Although, there was some chylomicron increment, a significant increase in the level of VLDL determined by lipid electrophoresis excludes the possibility of severe LPL deficiency.

\section{DISCUSSION}

In the last decades, women had increasing access to graduation in professional schools, building careers, and using contraceptive methods worldwide ${ }^{[15]}$. This has led to the delay of first marriage and first child birth. In fact, the average age at first birth rose from 22.7 years in 1980 to 28.2 years in $2013^{[16]}$. Indeed, many women are choosing to delay attempts to conceive to their thirties and forties, when they have a significantly higher risk of type 2 diabetes, metabolic syndrome and other non-communicable diseases causing lipid abnormalities. Furthermore, the proportion of women of reproductive age who are overweight or obese is also increasing at an alarming rate ${ }^{[17]}$. The incidence of severe hypertriglyceridemia in pregnant women might be higher than we believe.

Although during pregnancy measurement of lipid parameters is not performed routinely, in case of a lipemic blood sample, suggestive patient history or previous pancreatitis, determination of triglyceride level is definitely required. 


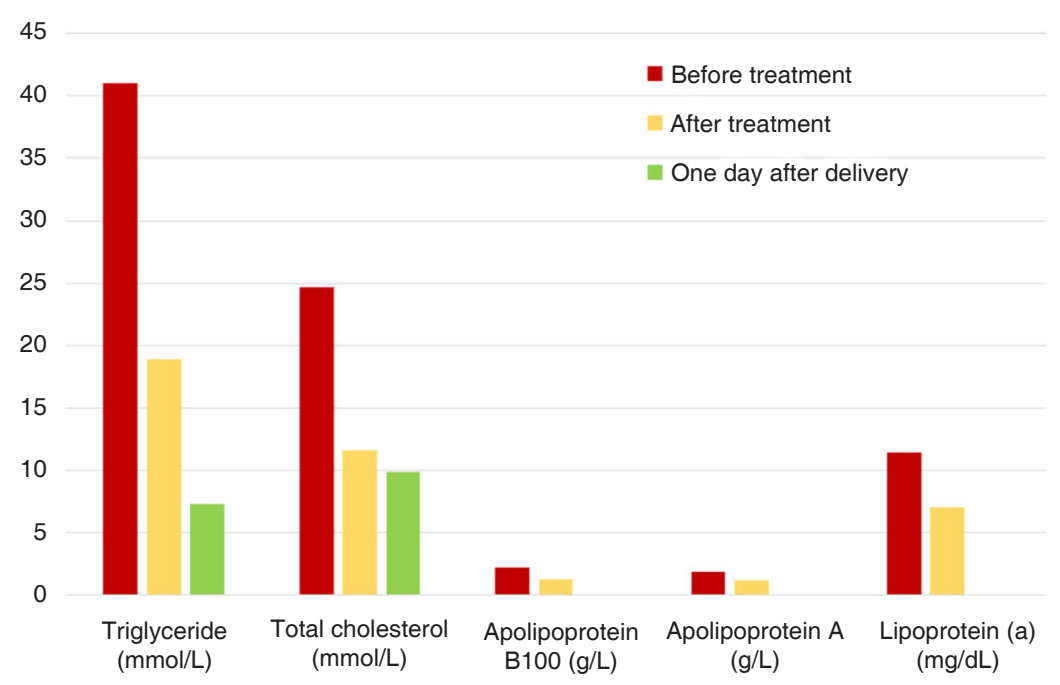

Figure 1. Lipid parameters before and immediately after treatment plasmapheresis, and one day after delivery

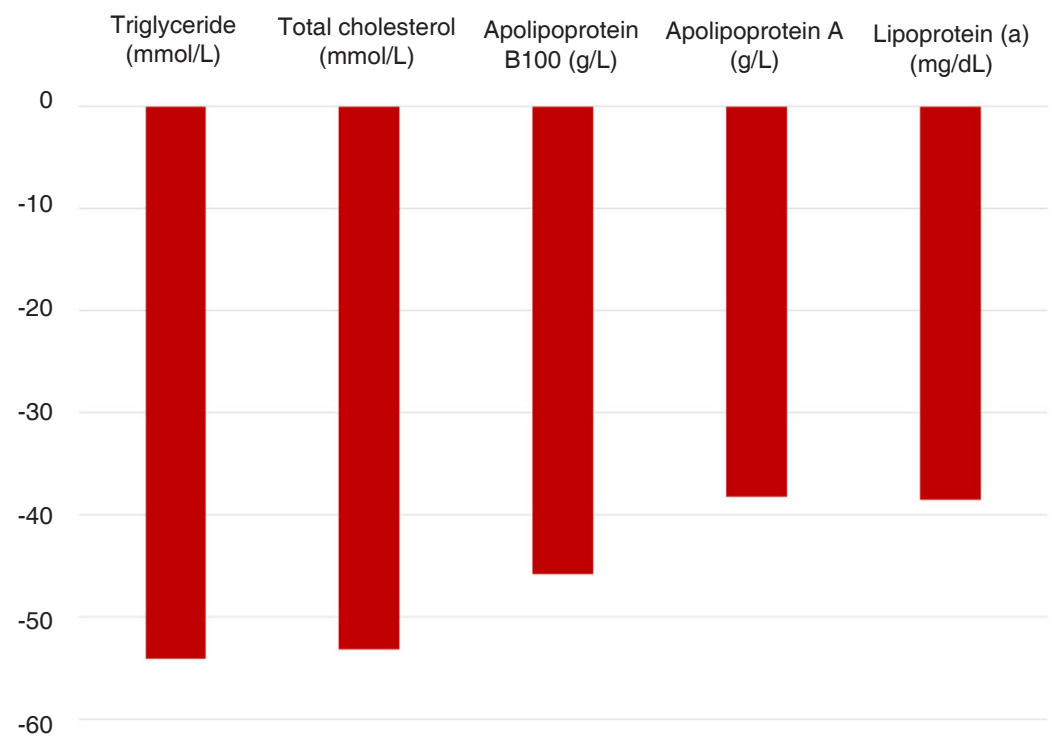

Figure 2. Changes (\%) in lipid parameters before and immediately after plasmapheresis treatment

While treatment of gestational hypertriglyceridemia-induced pancreatitis with plasmapheresis is welldefined ${ }^{[6]}$, the timing and frequency of plasmapheresis procedures in gestational hypertriglyceridemia without pancreatitis should be determined individually, depending on patient's history, symptoms, duration of pregnancy and the lowest triglyceride level available with diet. To date, there is no available guideline for the management of severe hypertriglyceridemia that develops during pregnancy. Although some case reports and series were published ${ }^{[12]}$, including treatment methods such as intravenous insulin and glucose, heparin and apheresis, they are not comparable, therefore, the clinician has to come to the optimal decision. The timing of the extracorporeal treatment can be an especially tender spot, since all of these procedures may provoke adverse events such as hemodynamical instability, which can be especially critical in late pregnancy. Early plasmapheresis as a successful treatment in hypertriglyceridemia-induced acute pancreatitis in firsttrimester pregnancy following in vitro fertilization was reported previously ${ }^{[18]}$. Here, we present a successful treatment in late pregnancy without adverse events. 
Regular check-up is highly recommended after delivery, during lactation and especially during subsequent pregnancies. Close cooperation between endocrinologist or lipid specialist and gynaecologist is also necessary. With the exception of lipoprotein lipase deficiency, primary hypertriglyceridemia disorders usually present in adulthood. Indeed, dozens of causative genetic abnormalities were described in severe hypertriglyceridemia. Genetic testing is available for suspected cases of familial chylomicronemia syndrome and dysbetalipo-proteinemia, but is not necessary for treatment ${ }^{[5]}$. Still, sequencing of candidate genes may help to guide future individualized therapeutic strategies in order to prevent further complications and to identify affected relatives using cascade screening.

In case of significantly increased values, plasmapheresis is a fast, effective and safe method for decreasing triglyceride level even in late pregnancy. However, multicentre, prospective studies including a larger number of participants are required to support the observations and to define novel therapeutic guidelines.

\section{DECLARATIONS}

\section{Authors' contributions}

Collection of data: Zsíros N, Harangi M

Analysis and/or interpretation of data: Zsíros N, Harangi M

Writing (not revising) all or sections of the manuscript: Zsíros $\mathrm{N}$

Manuscript review: Kovács B, Harangi M

Supervision: Paragh G, Balla J

Study design: Paragh G, Harangi M

\section{Availability of data and materials}

The clinical and laboratory data used to support the findings of this work are included within the article.

\section{Financial support and sponsorship}

This research was supported by the GINOP-2.3.2-15-2016-00005 project. The project is co-financed by the European Union under the European Regional Development Fund.

\section{Conflicts of interest}

All authors declared that there are no conflicts of interest.

\section{Ethical approval and consent to participate}

Informed consent was obtained from the patient after the approval of the local ethics committee.

\section{Consent for publication}

Informed consent was obtained from the patient.

\section{Copyright}

(c) The Author(s) 2019.

\section{REFERENCES}

1. Butte NF. Carbohydrate and lipid metabolism in pregnancy: normal compared with gestational diabetes mellitus. Am J Clin Nutr 2000;71:1256S-61.

2. Herrera E, Amusquivar E, López-Soldado I, Ortega H. Maternal lipid metabolism and placental lipid transfer. Horm Res 2006;65:59-64.

3. Brizzi P, Tonolo G, Esposito F, Puddu L, Dessole S, et al. Lipoprotein metabolism during normal pregnancy. Am J Obstet Gynecol 1999;181:430-4.

4. Knopp RH, Warth MR, Charles D, Childs M, Li JR, et al. Lipoprotein metabolism in pregnancy, fat transport to the fetus, and the effects of diabetes. Biol Neonate 1986;50:297-317. 
5. Ma Y, Ooi TC, Liu MS, Zhang H, McPherson R, et al. High frequency of mutations in the human lipoprotein lipase gene in pregnancyinduced chylomicronemia: possible association with apolipoprotein E2 isoform. J Lipid Res 1994;35:1066-75.

6. Wong B, Ooi TC, Keely E. Severe gestational hypertriglyceridemia: a practical approach for clinicians. Obstet Med 2015;8:158-67.

7. Goldberg AS, Hegele RA. Severe hypertriglyceridemia in pregnancy. J Clin Endocrinol Metab 2012;97:2589-96.

8. Gallos ID, Sivakumar K, Kilby MD, Coomarasamy A, Thangaratinam S, et al. Pre-eclampsia is associated with, and preceded by, hypertriglyceridaemia: a meta-analysis. BJOG 2013;120:1321-32.

9. Tang SJ, Rodriguez-Frias E, Singh S, Mayo MJ, Jazrawi SF, et al. Acute pancreatitis during pregnancy. Clin Gastroenterol Hepatol 2010;8:85-90.

10. Eapen DJ, Valiani K, Reddy S, Sperling L. Management of familial hypercholesterolemia during pregnancy: case series and discussion. J Clin Lipidol 2012;6:88-91.

11. Sivakumaran P, Tabak SW, Gregory K, Pepkowitz SH, Klapper EB. Management of familial hypertriglyceridemia during pregnancy with plasma exchange. J Clin Apher 2009;24:42-6.

12. Basar R, Uzum AK, Canbaz B, Dogansen SC, Kalayoglu-Besisik S, et al. Therapeutic apheresis for severe hypertriglyceridemia in pregnancy. Arch Gynecol Obstet 2013;287:839-43.

13. Piolot A, Nadler F, Cavallero E, Coquard JL, Jacotot B. Prevention of recurrent acute pancreatitis in patients with severe hypertriglyceridemia: value of regular plasmapheresis. Pancreas 1996;13:96-9.

14. Alberti KG, Zimmet PZ. Definition, diagnosis and classification of diabetes mellitus and its complications. Part 1: diagnosis and classification of diabetes mellitus provisional report of a WHO consultation. Diabet Med 1998;15:539-53.

15. Group ECW. Social determinants of human reproduction. Hum Reprod 2001;16:1518-26.

16. Steiner AZ, Jukic AM. Impact of female age and nulligravidity on fecundity in an older reproductive age cohort. Fertil Steril 2016;105:1584-8.e1.

17. Callaway LK, Prins JB, Chang AM, McIntyre HD. The prevalence and impact of overweight and obesity in an Australian obstetric population. Med J Aust 2006;184:56-9.

18. Reper P, Attou R, Gucciardo L, Gottignies P, Devriendt J, et al. Early plasmapheresis as a successful treatment in hypertriglyceridemiainduced acute pancreatitis in first trimester pregnancy following in vitro fertilization. Eur J Obstet Gynecol Reprod Biol 2014;179:257-8. 